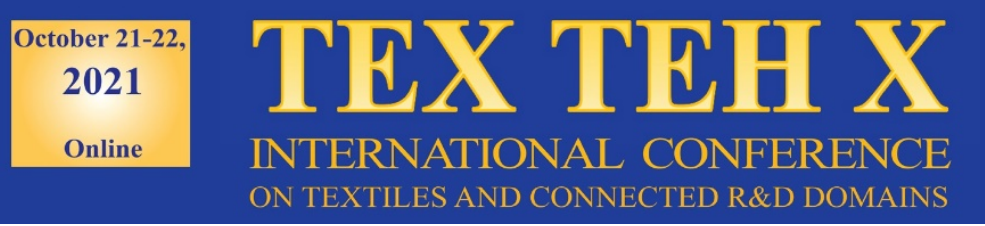

\title{
OVERVIEW OF THE KNITTED MATERIALS WITH VIBRATIONS DAMPING CAPACITY
}

\author{
DOI: 10.35530/TT.2021.54
}

\author{
C. Grosu ${ }^{* 1,2}$, M. Blaga ${ }^{1}$
}

1 “Gheorghe Asachi” Technical University, Doctoral School, Romania

${ }^{2}$ National Research Development Institute for Textiles and Leather, Romania

(E-mail: cristina.grosu@academic.tuiasi.ro; mirela.blaga@academic.tuiasi.ro)

\begin{abstract}
Textile materials are often subjected to different stresses, acting on them in two phases: during the knitting phase, when the yarns and the obtained structure are subjected to cyclic stress, but also during the use phase, when the knitted structures are subjected to various stresses. The dynamic behaviour of knitted fabrics in a vibrating environment is usually evaluated by standardized methods, such as the method using vibration exciters (e.g., ISO 10819:2013). However, in recent years, the authors' collective has carried out research to characterize the behaviour of knitted structures in a vibrating environment, using a well-known method for generating vibrations by impact excitation, which is specific to the mechanical field but also has a high potential for application in the textile field. This method refers to the determination of the free vibrations of an elastic system. Its measurement in the design phase of the system is considered a crucial step, since by knowing the frequency range of the system, the resonance phenomenon in the operational phase can be avoided. Similar results obtained by applying standardized methods for measuring vibration transmissibility and the currently adapted method from the field of mechanics to the field of textiles, represent a validation for this type of investigation process and also show the high potential of knitwear to be used in the vibration environment.
\end{abstract}

Keywords: free vibrations, knitted structures, dynamic excitation, structural parameters

\section{INTRODUCTION}

Although exposure to vibration can have very different causes, a major source of exposure is the use of work equipment that emits transmitted vibrations to the hand-arm system (HAV) or the whole body (WBV).

Depending on the characteristic parameters of the transmitted vibrations, such as frequency and amplitude, but also on secondary factors, such as the duration of exposure or the ambient temperature, vibration exposure can cause clinical manifestations that endanger the health of the worker, such as kinetosis, dizziness, vomiting or even inflammation of the tendons and muscles, leading in rare cases to joint swelling, reduced mobility and gangrene of the fingers.

Any workplace where workers are exposed to vibration must implement a very strict policy to prevent and reduce the risks of exposure to HAV and WBV. Personal protective equipment (PPE) is the final barrier between the exposed person and the risk and is the ultimate solution to protect against risks at the workplace.

To provide the highest level of protection against vibration, PPE must be able to absorb energy efficiently and maintain an optimal level of rigidity to avoid crushing it, in 
the event of an impact. It must also be of an acceptable thickness to allow handling of vibrating instruments without compromising dexterity and sense of touch [1].

\section{MANUFACTURING METHODS OF SPACER KNITTED FABRICS}

Spacer fabrics are one of the most widely used materials for engineering applications and the most widely used option for the manufacture of anti-vibration materials. Spacer fabrics (sandwiches) are complex three-dimensional constructions created by knitting two separate layers, joined by yarn segments or other knitted layers, in different bond ratios.

3D fabrics have great potential to replace commonly used materials as protective layers in PPE construction due to their high flexibility, good pressure recovery, and high tensile, flexural, and impact strength. Compared to most of the materials used, such as polyurethane, foam or rubber, they are more environmentally friendly, not easily flammable, have superior thermal comfort properties and also a low mass to volume ratio.

The structure of the outer layers, as well as the way in which the layers are bonded and the distance between them, offer a variety of possibilities for designing spacer fabrics with different physical-mechanical properties. Structures with outer layers made of the same raw material as well as structures with layers made of different raw materials can be produced [2].

Spacer knitted fabrics produced on warp knitting machines can be rigid or elastic structures, with open or closed stitches, this technology being by far the most inventive system for obtaining textiles. Double needle bar raschel knitting machines have the ability to produce the two outer layers on the front and back needle bars simultaneously with the process of feeding the binding yarns on these two needle bars to achieve the joining of the outer layers [3]. The 3D fabrics produced in this way are characterized by a very large volume with relatively low mass and offer high air permeability. The thickness of the fabrics ranges from 1.5 to $10 \mathrm{~mm}$, depending on the spacing of the needle bars, and can be up to 60 $\mathrm{mm}$ for special applications.

Spacer knits made on weft machines can be made on both, flat knitting machines equipped with two needle bars and circular machines equipped with a cylinder and disc. While spacer fabrics joined by binding threads can be produced on both, warp and weft knitting machines, spacer fabrics joined by layers can only be produced on weft knitting machines, the basic principle being similar to that of sandwich structures joined by binding threads. The knitting of the two outer layers begins independently on the two needle bars and at a certain point in time this process stops and the knitting of the connecting layers starts. After the knitting of the connecting layer is completed, the knitting of the independent outer layers starts again.

\section{EVALUATION OF THE SPACER KNITTED MATERIALS}

\subsection{Knitted materials}

The dynamic behaviour of different variants of knitted structures produced by specific technologies for warp and weft spacer fabrics was analysed under the influence of vibrations. A wide range of raw materials was used, such as cotton, polyamide, polyester, polypropylene and polyacrylonitrile.

The raw materials from which the spacer fabrics with the best anti-vibration properties were obtained are polyamide and polyester with the below characteristics, according to the specific manufacturing process, as follows: 


\section{Spacer warp knits:}

- for the outer layers: Polyester multifilament, in the fineness range 44-2500 dtex, with a number of filaments between 2 and 144 or polyamide multifilament, in the fineness range 44-167 dtex, with a number of filaments between 3 and 24;

- $\quad$ for the connecting yarns: Polyester monofilament, in the fineness range 33-680 dtex or polyamide monofilament, in the fineness range 22-33 dtex.

- $\quad$ the samples subjected to be evaluated, are represented in figure 1.

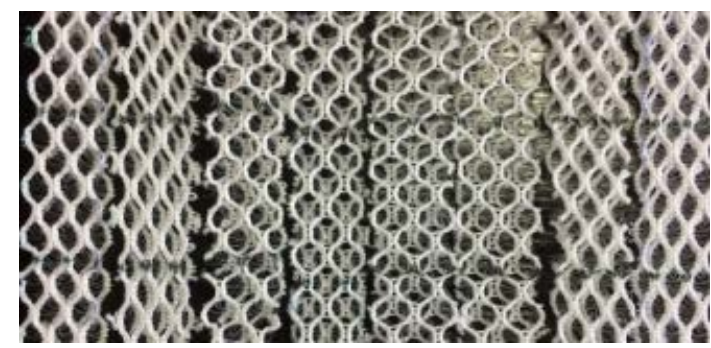

Fig. 1. Variants of spacer warp knitted structures [4]

\section{Spacer weft knits:}

- for the outer layers: Polyester/Elastan multifilament yarns, in different fineness range;

- for the connecting yarns: Polyester monofilament, in diameter range $0.13 \mathrm{~mm}$ $0.3 \mathrm{~mm}$;

- the needles sections of proposed knitted structures to be evaluated, are represented in figure 2 .
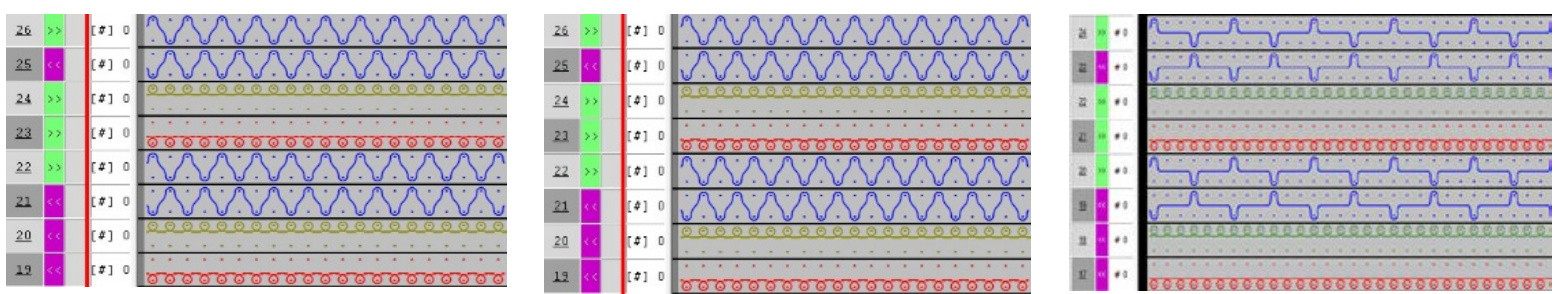

Fig. 2. Variants of spacer weft knitted structures

\subsection{Testing method}

PPE, such as anti-vibration gloves, must be marked with the symbol CE indicating that they have been tested and meet the requirements of the standard SR EN ISO 10819: 2013 - Mechanical vibration and shock - Hand-arm vibration - Measurement and evaluation of vibration transmissibility of gloves at the palm. The standard uses the method of vibration generation by excitation using a system equipped with a special handle to measure the clamping force and a device to measure the supply force of the system. A major disadvantage of this standard is that the measurement is only carried out in the area of the palm in the direction of the forearm.

The method proposed by the authors is based on the generation of vibrations by shock excitation to determine the natural frequencies of an elastic system, which plays a crucial role in avoiding, from the design stage, the possibility of resonance during use. This method is specific to the mechanical field and for its application it is necessary to use a specially designed test apparatus and carry out the following steps:

The application of the method refers to the excitation process of the elastic system, which consists of a metallic mass fixed to the knitted structure, to which an accelerometer is 
attached. A piezoelectric impact hammer is used as the exciter. The vibrations are exerted and measured in three directions: transverse, longitudinal and vertical.

Data analysis is performed by characterizing the movements of the metal mass attached to the knitted fabric. The signal is processed by a data acquisition system, and the Fast Fourier Transform (FTT) decomposes the signal and allows the frequency spectrum of a data sequence to be calculated.

The evaluation of the results consists in the characterization of the dynamic vibration behaviour for the tested knitted variants and the interpretation of the obtained responses in correlation with different structural parameters of the knitted fabric.

\section{PARAMETERS RELATED TO THE FABRICS BEHAVIOUR AT VIBRATIONS}

\subsection{Direction of producing and measuring vibration}

The recorded frequencies showed comparable vibration responses in the coursewise and walewise directions, while significantly higher values of natural frequencies were recorded in the perpendicular direction of the test. Therefore, a first conclusion is that the perpendicular direction is recommended as a possible final application for vibration isolation [5].

\subsection{Spacer fabrics properties with influence on the behaviour in vibration environment}

The results obtained indicated that the properties of the spacer knitted fabrics with the strongest impact on vibration behaviour, proved to be:

\section{Spacer yarn threading}

The tests showed a higher stability of the knit, both, in the case of the comparative evaluation between two knits, fed with one yarn per needle, respectively two yarns per needle, in the same ratio of 1:5, for the knit with double number of fed yarns on a needle, but also in the case of two knits, fed with a single thread per needle, but in a ratio of 1:3 and $1: 5$, respectively.

In this second case, the knitted structure with a higher number of connecting yarns, the one in the ratio of 1:3, showed a higher rigidity, implicitly a better vibration behaviour, which led to the conclusion that a higher number of connecting yarns positively influences the resistance of the knit to vibration, by increasing its rigidity $[1,6]$.

\section{Spacer yarn diameter}

The spacer yarns offer a better stability and structural balance as well as a positive effect on mechanical properties of the fabric.

Tests have shown that an insignificant increase of $0.02 \mathrm{~mm}$ in the spatial yarn diameter, significantly influences the behaviour of the knit, which increases its mass by about $15 \%$. This consequently implies a higher stiffness of the knit, demonstrated by higher values of natural frequencies $[1,6]$.

It can be hypothesized that the thicker the spacer yarn, the more rigid the knit will be, higher loading capacity will possess and implicitly higher values of natural frequencies will record.

\section{Yarn consumption}

From a range of 16 knitted structures, those made with the lowest yarn consumption, presented the lowest values of natural frequencies, implicitly the lowest level of rigidity. The variants made with the highest yarn consumption have registered the highest values of 
natural frequencies and implicitly due to the increased mass they present a higher rigidity, which will lead to a much better response in vibration environment [7].

\section{Fabric thickness}

Tests have shown that the thickness of the knit directly influences the values of natural frequencies, so that the thicker the knit, the higher the values of natural frequencies, implicitly a higher rigidity. To validate these results, a second method was applied, specific to the textile field, to determine the flexibility of knitwear, which validated the ranking of knitwears stiffness, based on the method of determining free vibrations [4].

\section{Raw materials of the outer layers}

Tests with several knitting variants, in which the structure of the fabric and the diameter of the spacer yarns were maintained, but the type of yarns for the outer layers was varied, showed that polyester and polyamide were the most recommended raw materials for the outer layers, with extremely small differences registered between them at the maximum local stress, less than 0.1 MPa. The knitted fabrics made of synthetic fibres such as PES and PP showed higher natural frequencies than the knitted fabrics made of cotton and PNA, which reached similar values between them [5].

\section{CONCLUSIONS}

The study of the vibration behaviour of the knitted fabric using the method proposed by the authors to determine the natural frequencies is confirmed by the fact that similar results were obtained as in the literature, where the standardized method of generating vibrations by excitation was applied.

The application of existing methods led to the same conclusions regarding the influence of structural and physical-mechanical parameters of the knitted structures on the vibration behaviour, such as: type of raw material [3,8-11], thickness of the knitted fabric [3,12], yarn consumption and some particularities of the spacer yarn: threading type $[3,13]$, yarn diameter $[3,13]$.

Analysis of the recorded frequencies revealed comparable vibration responses in the longitudinal and transverse directions, while significantly higher values of natural frequencies were recorded in the perpendicular direction of the test. This suggests that this direction is the more suitable for vibration isolation [1-7].

\section{ACKNOWLEDGEMENTS}

This work is the result of the research activity for the doctoral thesis "Technical textiles for protection against vibrations", carried out by the doctoral student Grosu (Pavel) Cristina under the careful guidance of the doctoral supervisor, Prof. Dr. Habil. Eng. Blaga Mirela.

\section{REFERENCES}

[1] Blaga, M., Seghedin, N.E., Knitted Spacer Fabrics Behaviour at Vibrations, In: Journal of Textile Engineering \& Fashion Technology, 2017, 3, 2, 602-608, https://doi.org/10.15406/jteft.2017.03.00092

[2] Buhai, C., Cercetari privind utilizarea firelor din fibre speciale in realizarea produselor de imbracaminte din tricot, Iasi, 2013

[3] Sum, N.W., Development of anti-vibration glove with weft knitted spacer fabric, Hong Kong, 2013 
[4] Blaga, M., Harpa, R., Seghedin, N.E., Marmarali, A., Ertekin, G., Evaluation of the knitted fabrics stiffness through dynamic testing, In: Materials Science and Engineering. IOP Publishing, 2019, https://doi.org/10.1088/1757-899X/459/1/012033

[5] Seghedin, N.E., Blaga, M., Ciobanu, R., Weft knitted fabrics behaviour under dynamic testing., Autex 12th World textile Conference, Zadar, 2012

[6] Blaga, M., Seghedin, N.E., Ciobanu, A.R., Weft knitted spacer fabrics response to vibrations, Autex World Textile Conference, Bursa, 2014

[7] Blaga, M., Seghedin, N.E., Buhai, C., Chitariu, D., Dinamic testing of the warp knitted spacer fabrics, Autex World Textile Conference, Dresden, 2013

[8] Liu, Y., Hu, H., An Experimental Study of Compression Behavior of Warp-knitted Spacer Fabric, In: Journal of Engineered Fibers and Fabrics, 2014, 9, 2, 61-69

[9] Liu, Y., Hu, H., Finite element analysis of compression behaviour of 3D spacer fabric structure, In: International Journal of Mechanical Sciences, 2015, 94, 95, 244-259, https://doi.org/10.1016/j.ijmecsci.2015.02.020

[10] Liu, Y., Hu, H., Vibration isolation behaviour of $3 D$ polymeric knitted spacer fabrics under harmonic vibration testing conditions, In: Polymer Testing, 2015, 120-129

[11] Liu, Y., Hu, H., Long, H., Zhao, L., Impact compressive behavior of warp-knitted spacer fabrics for protective applications, In: Textile Research Journal, 2012, 773-788, https://doi.org/10.1177/0040517511433147

[12] Liu, Y., Hu, H., Vibration Isolation Performance of Warp-knitted Spacer Fabrics, Fiber Society Spring Conference, Hong Kong: Curran Associates, Inc., 2011, 63-64

[13] Chaoyu, C., Junli, C., Fengxin, S., Huijuan, Y., Zhaoqun, D., Analysis of the damping property of warp-knitted spacer fabrics under damped free vibration, In: Textile Research Journal, 2017, 790-799 